\title{
A PILOT SEARCH FOR POPULATION III SUPERNOVA CANDIDATES IN THE SPITZER/IRAC DARK FIELD
}

\author{
Mark I. Frost ${ }^{1,2}$, Jason Surace ${ }^{1}$, Leonidas A. Moustakas ${ }^{3}$, and Jessica Krick ${ }^{1}$ \\ ${ }^{1}$ Spitzer Science Center, MS 220-6, Caltech, Pasadena, CA 91125, USA \\ 2 Astronomy Center, University of Sussex, Brighton, England BN1 9QH, UK \\ 3 JPL/Caltech, 4800 Oak Grove Dr., MS 169-327, Pasadena, CA 91109, USA \\ Received 2008 November 12; accepted 2009 April 30; published 2009 May 22
}

\begin{abstract}
We have undertaken a systematic search for candidate supernovae from high-redshift Population III stars in a field that has been observed with repeated imaging on a cadence of 2-3 weeks over a 2.2 year baseline, the Spitzer/IRAC Dark Field. The individual epochs reach a typical $5 \sigma$ depth of $1 \mu$ Jy in IRAC Channel $1(3.6 \mu \mathrm{m})$. Requiring a minimum of four epochs coverage, the total effective area searched is $214 \operatorname{arcmin}^{2}$. The unprecedented depth and multi-epochal nature of these data make it ideal for a first foray to detect transient objects which may be candidate luminous pair-instability supernovae from the primordial metallicity first stars. The search was conducted over a broad range of timescales, allowing for different durations of the putative candidates' light-curve plateau phases. All candidates were vetted by inspection of the Spitzer imaging data, as well as deep Hubble Space Telescope/Advanced Camera for Surveys F814W imaging available over the full field. While many resolved-source objects were identified with Spitzer variability, no transient objects were identified that could plausibly be identified as high-redshift supernovae candidates. The resulting $95 \%$ confidence level upper limit is $23 \mathrm{deg}^{-2} \mathrm{yr}^{-1}$, for sources with plateau timescales under $400 /(1+z)$ days and brightnesses above $\sim 1 \mu \mathrm{Jy}$.
\end{abstract}

Key words: infrared: stars - supernovae: general

\section{INTRODUCTION}

Primordial metallicity Population III stars are thought to be the first luminous objects to form in the universe. Their formation marks the end of the cosmic dark ages and an important transition of the universe from a homogeneous state to a highly structured one. The UV photons produced by such stars at high redshifts are also thought to be at least partly responsible for re-ionizing the universe (Tumlinson \& Shull 2000; Bromm et al. 2001; Schaerer 2002, 2003). It is believed that the explosive events that mark the end state of such stars seed the intergalactic medium with heavy elements (Gnedin \& Ostriker 1997; Furlanetto \& Loeb 2003; Greif et al. 2007). Hence, studying these objects is of great importance in helping us to understand the high-redshift universe.

To date, the supernovae marking the deaths of first stars (Pop III SNe) at high redshift have not been observed (though see Woosley et al. 2007). We turn to theoretical modeling to gain a better understanding of the properties of such stars and when they might have existed. It is thought that Population III stars formed out of primordial abundance $\mathrm{H} / \mathrm{He}$ gas in low-mass dark matter halos. For primordial abundance stars it is expected that the explosion mechanism may drive not only "classical" supernovae, but also "hypernovae" for certain progenitor masses, driven through an electron-positron instability mechanism that results in explosive events with up to approximately 100 times greater luminosities (Umeda \& Nomoto 2002). Exactly when the epoch of the first stars began is still a matter of debate but estimates place it at $10<z<50$ (Wise \& Abel 2005). Thereafter, such objects could exist in primordial metallicity pockets even at relatively low redshifts, even $z \leqslant 2.5$ (e.g., Scannapieco et al. 2005; Tornatore et al. 2007). The primordial metallicity of these stars leads to inefficient cooling mechanisms through $\mathrm{H}_{2}$, leading to very high stellar masses and to a top-heavy initial mass function (IMF) with a large fraction of stars having $M_{\star}>100 M_{\odot}$ (Bromm et al. 1999, 2002; Abel et al. 2000,
2002) and possibly even greater masses (Bromm \& Loeb 2004; Omukai \& Palla 2003).

In this Letter, we describe a search for candidate supernovae from high-redshift Population III stars in the Spitzer/IRAC dark field. The target field is described in Section 2. In Section 3, we summarize the theoretical modeling used as a guide for detecting transient objects, which would include candidate Pop III SNe. Section 4 outlines the search methods used. In Section 5, we compare the Pop III SNe rate upper limits we find with expectations from the literature, and highlight how this type of search may be extended in the future. Where needed, we adopt $H_{0}=73 \mathrm{~km} \mathrm{~s}^{-1} \mathrm{Mpc}^{-1}, \Omega_{m}=0.27$, and $\Omega_{\Lambda}=0.73$.

\section{THE IRAC DARK FIELD}

The IRAC dark field is the dark current calibration target for the Infrared Array Camera (Fazio et al. 2004) onboard the Spitzer Space Telescope. It is an extragalactic field of very low background, in Spitzer's continuous viewing zone near the North Ecliptic Pole. This area is observed at the start and end of each IRAC observing campaign (2-3 weeks apart) since Spitzer's first light. For technical reasons anchored on the need for high-quality dark frames and on the normal precession of the observatory, there is only a modest overlap in the observed area on the sky from epoch to epoch, making for a positiondependent observed cadence in the time domain data.

The data used in this analysis are based on 128 distinct epochs over the first 2.2 years of Spitzer's operations. Each epoch is composed of multiple individual exposures at all of the available IRAC exposure times. The total full IRAC mosaic in the adopted data set has a total observing time of $>500 \mathrm{hr}$, and covers an approximately circular area, 20 arcmin in diameter. Each point in this mosaic typically has more than $10 \mathrm{hr}$ of total integration time, with a maximum of $\sim 80 \mathrm{hr}$ in the area of maximal overlap across epochs. It is classically confusion-limited in all four IRAC channels (3.6 $\mu \mathrm{m}, 4.5 \mu \mathrm{m}, 5.8 \mu \mathrm{m}$, and $8.0 \mu \mathrm{m})$. 
The IRAC source extraction was done using Source EXtractor (Bertin \& Arnouts 1996).

The unprecedented depth and multi-epochal nature of these data make it ideal for a first foray into trying to detect supernovae from the first stars. The field will, in fact, continue to be observed through the full extent of the Spitzer Warm Mission, eventually giving a baseline of around seven (and possibly 10) years. There is now a wealth of multiwavelength data available in the dark field including Chandra and Hubble Space Telescope (HST)/ Advanced Camera for Surveys (ACS) F814W imaging (Krick et al. 2008).

To find transient objects, a common method is to search for significant residuals when differencing registered timeseries observations. However, because the IRAC point-spread function is asymmetric and fairly complex, and indeed has a different orientation on the image of each epoch, it is difficult to distinguish candidate transient objects from artifacts in the difference images. Therefore, the search is conducted through cross-correlating catalogs of objects detected in each individual epoch. Each epoch of observation has a typical $5 \sigma$ depth of $1 \mu \mathrm{Jy}$, or $m_{3.6 \mu \mathrm{m}}(\mathrm{AB}) \sim 23.9$. The catalogs from all epochs were cross-matched with each other using a 1 arcsec radius, resulting in a master database with 31,492 sources, each of which had at least one detection in a single distinct epoch. This master database contains all the critical information for a transience search. For each object, the following are recorded: in which epochs the object is within the observed field and detected, and its flux density; in which epochs the object is within the observed field and not detected, and the known sensitivity at that position (based on the integration time).

If the spectral energy distributions of putative high-redshift Pop III SNe are above the measured detection limits for some period of time contained within the timespan of this survey, they will appear as transient objects. We explore the expectations from theory and the potential efficacy of our search in the next section.

\section{EXPECTATIONS FROM THEORY}

Massive Pop III stars with primordial metallicity are thought to be common at high redshift. Stars with $M_{\star}<140 M_{\odot}$ or $M_{\star}>260 M_{\odot}$ are thought to form black holes at the end of their evolution (e.g., Fryer et al. 2001; Heger \& Woosley 2002). Those which have masses between 140 and $260 M_{\odot}$ are thought to end their lives as pair-instability supernova (PISNe). Once helium burning in the core of such stars has ceased there is sufficient entropy to create positron-electron pairs (Wise \& Abel 2005). This process converts thermal energy to the mass of the particle pair and the pressure in the core is reduced. This leads to a partial collapse which triggers a thermonuclear explosion. The star is completely destroyed leading to a PISN, in which no remnant is left behind. At least one relatively local analog may already have been observed (SN 2006gy in NGC 1260; Smith et al. 2007; Woosley et al. 2007), which lends support to the possibility of this mechanism. PISNe would be "host-less" and as much as 100 times more luminous than more-typical supernovae.

To estimate the chances of detecting such objects, we turn to predictions from the literature for anticipated luminosities, durations (through their light curves), and frequency of events. Scannapieco et al. (2005), using light curves calculated by Weinmann \& Lilly (2005), predict peak apparent magnitudes of $m_{\mathrm{AB}} \sim 26.8$ at $z=10$ for $250 M_{\odot}$ PISN, assuming negligible extinction. This suggests that the typical by-epoch depth of
Table 1

Predicted Population III Supernova Rates Found in the Literature

\begin{tabular}{lccc}
\hline \hline $\begin{array}{l}d N / d z \\
\left(\mathrm{deg}^{-2} \mathrm{yr}^{-1}\right)\end{array}$ & $\begin{array}{c}M_{\text {progenitor }} \\
\left(M_{\odot}\right)\end{array}$ & $z$ & Reference \\
\hline$\sim 0.2$ & 250 & $\sim 10$ & Heger et al. (2002) \\
0.34 & $100-500$ & 10 & Wise \& Abel (2005) \\
50 & 250 & $>15$ & Mackey et al. (2003) \\
11 & 100 & $>13$ & Cen (2003) \\
25 & $140-260$ & 5 & Weinmann \& Lilly (2005) \\
\hline
\end{tabular}

Notes.

a Rate of Heger et al. (2002) incorporating correction factor determined by Weinmann \& Lilly (2005) and recalculated at $z=10$.

${ }^{\mathrm{b}}$ Corrected rate determined by Weinmann \& Lilly (2005).

$m_{3.6 \mu \mathrm{m}}(\mathrm{AB}) \sim 24$ of our search may be able to detect such objects at $z \sim 3-5$.

PISN light curves are calculated in Wise \& Abel (2005), Heger et al. (2002), and Scannapieco et al. (2005). A broad plateau phase is expected, which could last from $\sim 10$ days to as long as a full year in the frame of the event. Since in the observed frame the light curve is stretched by a factor of $(1+z)$, there could be events that would appear as near-continuous sources over the entire 2.2 year monitoring span of the current data set. As we ultimately anticipate a 7 to 10 year data set by the end of the Spitzer Warm Mission, there is great potential in future analyses to encompass more plateau-duration possibilities.

In Table 1, we list several predicted Pop III SNe differential rates from the literature. The rates quoted in Table 1 for Heger et al. (2002) and Cen (2003) incorporate the corrections determined by Weinmann \& Lilly $(2005)$ of $(1+z)^{-2}$ and $(1+z)^{-1}$, respectively. These rates are over redshift ranges largely beyond the sensitivity of our search, but are quoted here for completeness. By the review in Weinmann \& Lilly (2005), realistic rates are expected to be $d N / d z \sim 4 \mathrm{deg}^{-2} \mathrm{yr}^{-1}$ for $z>15$ and $0.2 \mathrm{deg}^{-2} \mathrm{yr}^{-1}$ for $z>25$. Wise \& Abel (2005) find a Pop III SNe rate of $0.34 \mathrm{deg}^{-2} \mathrm{yr}^{-1}$ at $z=10$, which changes negligibly over the mass range $100 M_{\odot}<M_{\star}<500 M_{\odot}$. A wide range of values are expected, $\sim 0.2-50 \mathrm{deg}^{-2} \mathrm{yr}^{-1}$, which is indicative of how the parameters involved in such predictions are still not well constrained. We also refer the reader to Scannapieco et al. (2005) for further discussion of these predictions.

\section{SEARCH METHOD}

The search for transient objects is based on the master database described in Section 2. Systematic searches were conducted for three different ranges of possible plateau durations (in the observed frame), which are appropriate to the 2.2 year baseline of the present data set, and which are plausible based on some of the theoretical expectations for the light curves. These are $0<t_{\mathrm{obs}}<100,100<t_{\mathrm{obs}}<200$, and $200<t_{\mathrm{obs}}<400$ days. In addition to the practical convenience of analyzing these data in this compartmentalized fashion, it also anticipates the possibility that some transient-source light curves may be truncated by either end of our 2.2 year baseline by different timescales.

We remind the reader that the light curves of all sources are largely discontiguous because the precise pointing (and orientation) varies epoch by epoch. This also affects the precise depth of each epoch, and changes the on-sky orientation of the IRAC diffraction pattern essentially leading to rotating diffraction spikes across images. We find that the photometry of 
objects near bright stars is systematically contaminated by these rotating diffraction spikes. Therefore, we identify the stars and the sources close enough to them to be affected, and explicitly remove them from our master catalog. Imaging with $H S T / A C S$ F814W was obtained between 2006 November and December, one year after the end of the IRAC data set described here. With this data, a plot of the Source EXtractor (Bertin \& Arnouts 1996) ISOAREA value versus aperture magnitude photometry clearly separates the ridge of unresolved sources from resolved galaxies, because unresolved sources have smaller ISOAREA than galaxies at any given magnitude. This method identifies 1147 unresolved sources. We empirically determine a radius of $r=0.06 \times S_{3.6 \mu \mathrm{m}}$ arcsec (but no greater than 30 arcsec), to mask out a circular area around each star also removing an additional 5858 sources which were in close proximity.

Given the relative nonhomogeneity in the survey's depth and cadence sampling, we apply several criteria to remove erroneous sources. (1) We impose an upper flux density limit of $40 \mu \mathrm{Jy}$, which is an empirically determined practical threshold for removing additional objects that are affected by diffraction spike artifacts, beyond the masked radii, and object de-blending issues. (2) For all sources we only include epochs which have a $5 \sigma$ detection of $>1 \mu \mathrm{Jy}$. (3) We do not consider sources that appear in only a single epoch. (4) Sources detected in only two or three epochs are only included in our search if they have $5 \sigma$ detections of $>1 \mu \mathrm{Jy}$ in all epochs. (5) For any object with multiple epoch detections, we require the $3.6 \mu \mathrm{m}$ photometric uncertainty to be less than $10 \%$ in at least half of its detected epochs. This ensures that this source is not rejected on the basis of large uncertainties in a small number of epochs (e.g., due to some epochs being particularly shallow relative to the others). (6) Finally, we require at least two significant nondetections to ensure a clear transient signal.

After the application of each of these criteria, 650 candidates remained that warranted more careful follow-up. The $3.6 \mu \mathrm{m}$ light curves and the corresponding IRAC and HST imaging were all visually inspected by custom-built software that clearly identified all salient aspects for the object in the master database. No a priori restrictions were placed on the shape of the light curve. Several objects with otherwise flat light curves were found to have a dramatic "flare-up" in a single epoch. Careful inspection of the individual IRAC $100 \mathrm{~s}$ exposures of that epoch showed the spike to be a cosmic ray incident. The remaining candidates were found to have resolved-source counterparts in the HST data, and are therefore candidate low-redshift active galactic nuclei, which though extremely interesting in their own right, are clearly not Pop III SNe candidates. At the end of this careful analysis and vetting procedure, no viable candidates survived. In the next section we calculate the formal limits implied by our search.

\section{DISCUSSION AND CONCLUSIONS}

No Pop III SNe candidates to our sensitivity limit of $m_{3.6 \mu \mathrm{m}}(\mathrm{AB}) \sim 24$ were identified. For the areal search over a total of $214 \mathrm{arcmin}^{2}$, the rate limit is below $8 \mathrm{deg}^{-2} \mathrm{yr}^{-1}$. Using the same area but also modeling this nondetection limit as a Poisson distribution, the $95 \%$ confidence upper limit is $23 \mathrm{deg}^{-2} \mathrm{yr}^{-1}$, ignoring cosmic variance uncertainties. At $z \leqslant[3,5,10]$ this also corresponds to a volumetric rate of $[910,480,270] \mathrm{Gpc}^{-3} \mathrm{yr}^{-1}$, respectively. These limits are approximate, as the precise survey area relevant to each individual source is a complicated function of the varying field and depth by epoch. Furthermore, these limits only apply to moderate-duration events, with plateau phases lasting less than $\sim 400 /(1+z)$ days, by construction of the search. Many of the predicted light curves given, e.g., by Scannapieco et al. (2005) have plateau phases up to one year in the rest frame, which would be too long in the observed frame to be detected by the present survey, but which may be approachable in future incarnations of the Spitzer Dark Field surveys.

The Wise \& Abel (2005) and Heger et al. (2002) $z=10$ predicted differential rates of $d N / d z \sim 0.34 \mathrm{deg}^{-2} \mathrm{yr}^{-1}$ and $\sim 0.2 \mathrm{deg}^{-2} \mathrm{yr}^{-1}$, respectively, are not ruled out, even if they appear at lower redshifts which may be above our sensitivity limit. Given that our search is most effectively probing $z \sim 3-5$, the differential rate of Weinmann \& Lilly (2005) of $25 \mathrm{deg}^{-2}$ $\mathrm{yr}^{-1}$ at $z=5$ is broadly comparable to our limit. It should be noted the very luminous PISNe that we would be sensitive to in this survey may be only a small fraction of all high-redshift supernova events. There is a distinction currently being made between Pop III.1 and III.2 stars, where the former class are of fully primordial abundance, and form in dark matter mini-halos, resulting in the pre-requisite stellar masses of above $\sim 100 M_{\odot}$ to produce PISNe (Johnson \& Bromm 2006; McKee \& Tan 2008). These are distinct from the Pop III.2 stars, which are expected to form through atomic cooling processes, producing only $\sim 10 M_{\odot}$ progenitors (Greif \& Bromm 2006). The PISNe Pop III.1 progenitors may consist of only some $10 \%$ of Pop III SNe (Greif \& Bromm 2006). Furthermore, the "pristine" Pop III.1 progenitors can suffer dramatic negative feedback (e.g., McKee \& Tan 2008), which may additionally limit their relative numbers. These were all considerations not yet taken in the predictions by Mackey et al. (2003), which led to the high expectation rates.

To do a comprehensive search for Pop III SNe using Spitzer (or a future platform with similar capabilities) would require a survey area of $1 \mathrm{deg}^{2}$ with exposures of $5000 \mathrm{~s}(50 \times 100 \mathrm{~s}$ exposures) per point on the sky. This would reach $m_{\mathrm{AB}} \sim 26$ at the 3-5 $\sigma$ level, depending on extinction. Each epoch would require approximately $200 \mathrm{hr}$ of observation to cover the survey area with a 5 arcmin field of view. This greater depth complicates the IRAC data reduction as confusion is a significant, but tractable problem. Trading depth for greater area may not be optimal for detecting the highest redshift Pop III SNe, which are expected to be quite faint, $m_{\mathrm{AB}} \gtrsim 26$. This requirement is increasingly relaxed for lower redshift events, which may possibly exist at redshifts as low as $z \sim 2$. This ideal survey would need to be imaged every two months for a period of several years in order to plausibly sample much or all of the plateau phase of the light curve. The total program would therefore be of the order of 2000-3000 hr.

In the meanwhile, the current data set continues to expand as Spitzer continues to operate, acquiring new IRAC observations every 2-3 weeks. Including observations made during the Spitzer Warm Mission, the full data set will span at least seven years, and maybe as many as 10 full years. Future searches following the procedure described here should be powerful for identifying plausible candidates, or at least in setting firmer limits on the production rate of PISNe at high redshift, and thus setting practical limits on the relative abundances of Pop III.1 versus Pop III.2 progenitors ultimately informing studies of reionization of the high-redshift universe.

Support for this work was provided by NASA through the Spitzer Space Telescope Visiting Graduate Student Program, through a contract issued by the Jet Propulsion Laboratory, 
California Institute of Technology under a contract with NASA. The work of L.A.M. was carried out at the Jet Propulsion Laboratory, California Institute of Technology, under a contract with NASA. This research has made use of data from the Spitzer Space Telescope, which is operated by the Jet Propulsion Laboratory, California Institute of Technology under a contract with NASA, and the NASA/ESA Hubble Space Telescope, obtained at the Space Telescope Science Institute, which is operated by the Association of Universities for Research in Astronomy, Inc., under NASA contract NAS 5-26555. These observations are associated with program No. 10521. Support for program No. 10521 was provided by NASA through a grant from the Space Telescope Science Institute, which is operated by the Association of Universities for Research in Astronomy, Inc., under NASA contract NAS 5-26555. Support for this work was also provided by STFC studentship PPA/S/S2005/04270. L.A.M. is grateful to Tom Abel and Ranga-Ram Chary for many discussions on this topic. We also thank Seb Oliver for his useful comments and suggestions. We are grateful to the anonymous referee for comments that have helped focus and improve this report.

\section{REFERENCES}

Abel, T., Bryan, G. L., \& Norman, M. L. 2000, ApJ, 540, 39 Abel, T., Bryan, G. L., \& Norman, M. L. 2002, Science, 295, 93 Bertin, E., \& Arnouts, S. 1996, A\&AS, 117, 393

Bromm, V., Coppi, P. S., \& Larson, R. B. 1999, ApJ, 527, L5
Bromm, V., Coppi, P. S., \& Larson, R. B. 2002, ApJ, 564, 23

Bromm, V., Kudritzki, R. P., \& Loeb, A. 2001, ApJ, 552, 464

Bromm, V., \& Loeb, A. 2004, New Astron., 9, 353

Cen, R. 2003, ApJ, 591, 12

Fazio, G. G., et al. 2004, ApJS, 154, 10

Fryer, C. L., Woosley, S. E., \& Heger, A. 2001, ApJ, 550, 372

Furlanetto, S. R., \& Loeb, A. 2003, ApJ, 588, 18

Gnedin, N. Y., \& Ostriker, J. P. 1997, ApJ, 486, 581

Greif, T., \& Bromm, V. 2006, MNRAS, 373, 128

Greif, T., Johnson, J. L., Bromm, V., \& Klessen, R. S. 2007, ApJ, 670, 1

Heger, A., \& Woosley, S. E. 2002, ApJ, 567, 532

Heger, A., Woosley, S., Baraffe, I., \& Abel, T. 2002, in Proc. MPA/ESO/ MPE/USM Joint Astronomy Conf., ESO Astrophysics Symp., Lighthouses of the Universe: The Most Luminous Celestial Objects and Their Use for Cosmology, ed. M. Gilfanov, R. Sunyaev, \& E. Churazov (Berlin: Springer), 369

Johnson, J. L., \& Bromm, V. 2006, MNRAS, 366, 247

Krick, J. E., Surace, J. A., Thompson, D., Ashby, M. L. N., Hora, J. L., Gorjian, V., \& Yan, L. 2008, ApJ, 686, 918

Mackey, J., Bromm, V., \& Hernquist, L. 2003, ApJ, 586, 1

McKey, C. F., \& Tan, J. C. 2008, ApJ, 681, 771

Omukai, K., \& Palla, F. 2003, ApJ, 589, 677

Scannapieco, E., Madau, P., Woosley, S., Heger, A., \& Ferrara, A. 2005, ApJ, 633,1031

Schaerer, D. 2002, A\&A, 382, 28

Schaerer, D. 2003, A\&A, 397, 527

Smith, N., et al. 2007, ApJ, 666, 1116

Tornatore, L., Ferrara, A., \& Schneider, R. 2007, MNRAS, 382, 945

Tumlinson, J., \& Shull, J. M. 2000, ApJ, 528, L65

Umeda, H., \& Nomoto, K. 2002, ApJ, 565, 385

Weinmann, S. M., \& Lilly, S. J. 2005, ApJ, 624, 526

Wise, J. H., \& Abel, T. 2005, ApJ, 629, 615

Woosley, S. E., Blinnikov, S., \& Heger, A. 2007, Nature, 450, 7168 